\title{
Laboratory and clinical trials of cocamide diethanolamine lotion against head lice
}

Ian F Burgess, Elizabeth R Brunton, Christine M Brown

Context: During the late 1990s when insecticide resistance had rendered a number of treatment products ineffective some companies saw this as an opportunity to develop alternative types of treatment. We investigated the possibility that a surfactant-based lotion containing 10\% cocamide diethanolamine (cocamide DEA) was effective to eliminate head louse infestation. Settings and Design: Initial in vitro testing of the lotion formulation versus laboratory reared body/clothing lice followed by two randomised, controlled, community-based, assessor blinded, clinical studies. Materials and Methods: Preliminary laboratory tests were performed by exposing lice or louse eggs to the product using a method that mimicked the intended use. Clinical Study 1: Children and adults with confirmed head louse infestation were treated by investigators using a single application of aqueous $10 \%$ cocamide DEA lotion applied for 60 minutes followed by shampooing or a single $1 \%$ permethrin creme rinse treatment applied to pre-washed hair for 10 minutes. Clinical Study 2: Compared two treatment regimens using 10\% cocamide DEA lotion that was concentrated by hair drying. A single application left on for 8 hours/overnight was compared with two applications 7 days apart of 2 hours duration, followed by a shampoo wash. Results: The initial laboratory tests showed a pediculicidal effect for a 60 minutes application but limited ovicidal effect. A longer application time of 8 hours or overnight was found capable of killing all eggs but this differed between batches of test material. Clinical Study 1: Both treatments performed badly with only 3/23 (13\%) successful treatments using cocamide DEA and 5/25 (23.8\%) using permethrin. Clinical Study 2: The single overnight application of cocamide DEA concentrated by hair drying gave 10/56 (17.9\%) successes compared with 19/56 (33.9\%) for the 2 hour application regimen repeated after 1 week. Intention to treat analysis showed no significant difference $(p=0.0523)$ between the treatments. Over the two studies there were 18 adverse events possibly or probably associated with treatment, most of which were increased pruritus after treatment.

Conclusions: Cocamide DEA 10\% lotion, even when concentrated by hair drying, showed limited activity to eliminate head louse infestation. 
1 Laboratory and clinical trials of cocamide diethanolamine lotion against head lice.

2

\section{Abstract}

4 Context: During the late 1990s, when insecticide resistance had rendered a number of treatment

products ineffective, some companies saw this as an opportunity to develop alternative types of treatment. We investigated the possibility that a surfactant-based lotion containing $10 \%$ cocamide diethanolamine (cocamide DEA) was effective to eliminate head louse infestation.

Settings and Design: Initial in vitro testing of the lotion formulation versus laboratory reared body/clothing lice followed by two randomised, controlled, community-based, assessor blinded, studies.

Materials and Methods: Preliminary laboratory tests were performed by exposing lice or louse eggs to the product using a method that mimicked the intended use.

Study 1: Children and adults with confirmed head louse infestation were treated by investigators using a single application of aqueous $10 \%$ cocamide DEA lotion applied for 60 minutes followed by shampooing or a single $1 \%$ permethrin creme rinse treatment applied to pre-washed hair for 10 minutes.

Study 2: Compared two treatment regimens using $10 \%$ cocamide DEA lotion that was concentrated by hair drying. A single application left on for 8 hours/overnight was compared with two applications 7 days apart of 2 hours duration, followed by a shampoo wash.

Results: The initial laboratory tests showed a 60 minutes application gave a good pediculicidal and ovicidal effect. A longer application time of 8 hours or overnight was found capable of killing all eggs but this differed between batches of test material. Study 1: Both treatments performed badly with only $3 / 23$ (13\%) successful treatments using cocamide DEA and 5/25 (23.8\%) using permethrin.

Study 2: The single overnight application of cocamide DEA concentrated by hair drying gave 10/56 (17.9\%) successes compared with 19/56 (33.9\%) for the 2 hour application regimen repeated after 1 week. Intention to treat analysis showed no significant difference $(p=0.0523)$ between the treatments.

Over the two studies there were 18 adverse events possibly or probably associated with treatment, most of which were increased pruritus after treatment.

Conclusions: Cocamide DEA 10\% lotion, even when concentrated by hair drying showed limited activity to eliminate head louse infestation.

Ian F Burgess, Elizabeth R Brunton, Christine M Brown 
41 Insect Research \& Development Limited, Cambridge, UK

42

43 Corresponding author: Ian Burgess

44

45 Insect Research \& Development Limited, 6 Quy Court, Colliers Lane, Stow-cum-Quy, 46 Cambridge, CB25 9AU, UK.

47

48 Tel: +441223810070

49 Email: ian@insectresearch.com

50 


\section{Introduction}

52 Interest in the use of plant derived pediculicides, mostly essential oils, was rekindled following the 53 discovery of head lice resistant to insecticides in the 1990s (Veal, 1996; Yang et al., 2004;

54 Heukelbach, Speare \& Canyon, 2009). These developments also included non-volatile fixed 55 vegetable oils, with anecdotal claims of effectiveness for olive oil, mayonnaise, margarine, and coconut oil, which are messy to use and have doubtful effectiveness. Unmodified plant oils have been used for centuries as hair conditioners in southern Europe, South Asia, and Africa, without affecting lice as confirmed by laboratory tests of so-called "home remedies" (Takano-Lee et al., 2004). However, modified vegetable oil surfactants are widely used in toiletry cleansing products to remove oils and other materials from hair. Some of these may dissolve waterproofing lipids that protect the louse cuticle from dehydration but there has been little interest in evaluating them clinically for effectiveness.

Cocamide diethanolamine (cocamide DEA) is a surfactant that stabilises the foam in "stripping" or "clarifying" shampoos and is highly efficient at removing lipids and other deposits from the hair. It has been used in head louse treatments, mainly as an excipient but also an active ingredient, commonly described as modified coconut oil. During the early 1990s several "modified coconut oil" products were sold in Central Europe, but efficacy studies were poorly reported with unclear methodologies (Mülhofer, 1994). One manufacturer suggested the material asphyxiated lice by blocking the spiracles; assuming that coconut oil has an occlusive effect. In reality, this potent surfactant is more likely to disrupt the cuticular lipid of the lice.

We have conducted an investigation of the activity of cocamide DEA in the treatment of head louse infestation. Prior to initiating clinical studies the material was tested in vitro to confirm that the sponsor's formulation was active and to establish whether the approach to dosing, as set out in a previous report (Mülhofer, 1994), was appropriate. The initial randomised study was planned to show equivalence of the cocamide DEA $10 \%$ lotion with $1 \%$ permethrin creme rinse. However, this study was not successful so further in vitro tests were performed to see if a more effective treatment regimen was possible using this formulation. As a result of these tests two possible treatment options for the cocamide DEA preparation were selected and subsequently tested in a second clinical study. 
82

83 Methods and Materials

84 Pre-clinical studies

85 We performed pre-clinical laboratory evaluations of cocamide DEA in a manner intended to 86 mimic as closely as possible use of the product by a consumer in essentially the same way as 87 described previously for other products (Burgess, 1991; Burgess et al., 2012). We used laboratory 88 reared human clothing/body lice, Pediculus humanus humanus, to conduct the in vitro tests against adult insects. The lice were given squares of nylon gauze substrate throughout the tests. We provided adult lice with nylon gauze as a substrate for oviposition so we could handle the eggs without risk of damaging them and the effects of the treatment could be observed easily.

Before the first clinical study we performed tests to check the claims made previously by Mülhofer (1994) that the product was completely effective when applied for 60 minutes. In these tests we compared two potential formulations of aqueous cocamide DEA with $3.5 \%$ and $10 \%$ active substance, using three batches of 20 lice on $15 \times 15 \mathrm{~mm}$ squares of gauze as the test insects. In this test method we fully immersed the insects in the product for 10 seconds then, using forceps, lifted the gauze bearing the insects from the fluid, which allowed excess liquid to drain off as it would if the lotion had been poured onto the scalp. We then incubated them in closed $55 \mathrm{~mm}$ diameter plastic Petri dishes for 60 minutes, still wetted with the test fluid, followed by washing with non-medicated shampoo (Boots frequent wash shampoo), followed by rinsing and blotting dry. The lice were maintained overnight in a humidified incubator $\left(30^{\circ} \pm 2^{\circ}\right.$ Celsius and $50 \%$ relative humidity) and the effects of the treatment were recorded 24 hours after the initial exposure. For this first investigation freshly laid eggs were treated in the same way as lice, but in this case using only the $10 \%$ cocamide DEA mixture and then, after washing and rinsing, incubated at $30^{\circ} \pm 2^{\circ}$ Celsius and $50 \%$ humidity until the untreated control group of eggs had completed hatching, approximately 10 days later. In both louse and egg tests, the control groups were treated to the same procedures except we used tap water in place of the test formulation.

110 The evaluation of the effect of treatments on lice determined whether they were alive and moving 111 normally ("Mobile"); not walking but possibly exhibiting small movements of appendages or 112 peristalsis of the gut ("Immobile"); or immobile with no detectable sign of residual life ("Killed"). 
113 Both "Immobile" and "Killed" were included in mortality figures. For louse eggs, those that had

114 developed fully and from which a living louse nymph had emerged completely were recorded as

115 "Hatched". In some cases the nymph had developed and started the emergence process but had

116 died before escaping from the eggshell ("Half-hatched"). Eggs in which there was no obvious

117 sign of embryonic development, specifically no appearance of the eyespot, which would normally

118 become apparent between the third and fourth day of incubation, were designated "Undeveloped",

119 whereas eggs that contained an embryo with eyespots but that failed to hatch were recorded as

120 having "Died". In the case of louse eggs, both "Died" and "Undeveloped" were included in

121 mortality figures.

122

123 In preparation for the second clinical study we ran a series of tests against louse eggs, using

124 essentially the same methods, to compare different treatment regimens of exposure time (1 hour, 2

125 hours, or overnight); washing with shampoo followed by rinsing or washing with water only; and

126 the influence of hair drying over a period of approximately 5 minutes, using an electric hair dryer

127 on "Cool" setting held at a variable distance of approximately $30 \mathrm{~cm}$ from the test specimens,

128 compared with humidification after application of the product. The temperature during hair drying

129 was modulated by placing the fingers of the operator's hand between the air jet and the test insects

130 and by movement of the device nearer of further away during the drying process so that a

131 temperature of no greater than $40^{\circ}$ Celsius occurred in the dish where the insects were held,

132 measured using a thermocouple probe. Also we compared the sensitivity of louse eggs of different

133 ages to the product by collecting eggs on a single day, storing them under the same conditions and

134 then treating randomly sampled gauze squares, each bearing approximately 100 eggs, on the

135 subsequent test days. As with the first series of laboratory tests the control groups were treated in

136 the same way but using tap water for the initial exposure to fluid.

137

138 After completion of the second clinical study, the low level of efficacy achieved, despite using

139 longer application times and increasing the dose concentration of the cocamide DEA by

140 evaporation, caused us to consider the possibility that the two batches of cocamide DEA lotion

141 were different in some way. We were able to do this by performing further in vitro tests in which

142 different groups of louse eggs from the same batch were treated with samples the two batches of

143 lotion, using both 2 hour and overnight applications followed by a water rinse. 
145 Clinical studies

146 Study medications

147 We conducted two randomised, controlled, assessor blinded clinical studies, using essentially the 148 same procedures throughout, although the treatments offered were different. In both studies the 149 investigative product was a preserved aqueous solution of cocamide diethanolamine (cocamide

150 DEA) $10 \%$. This preparation was supplied in $100 \mathrm{~mL}$ polyethylene bottles with a pour-on

151 dispenser, applied systematically to saturate the hair and scalp, and massaged in. Investigators 152 applied all the treatments.

153

154 In the first study, one group of participants was randomised to receive cocamide DEA 10\% (batch number LI 35101) applied for 60 minutes followed by washing with non-medicated shampoo

156 (Boots frequent wash shampoo) then rinsing with water. The comparison group was treated using 157 1\% permethrin creme rinse (Lyclear creme rinse, Chefaro UK Ltd, Huntingdon, UK) applied for 15810 minutes to pre-washed and towel dried hair, followed by rinsing. Both products were applied 159 on a single occasion. Shampoo was supplied to both groups by the investigators applying the 160 treatment. No guidance on routine hair washing was given for this study.

162 The second study compared two treatment regimens using cocamide DEA $10 \%$ (batch number $\mathrm{NH}$ 163 35101). In one group we applied the product to dry hair until it was thoroughly soaked then 164 evaporated the excess water using a hair dryer leaving the hair sticky, with the appearance of 165 having been heavily oiled. The length of the "drying" process varied according to the length and 166 thickness of the hair and ranged from approximately 3-20 minutes, which was determined by the appearance and feel of the treated hair. This was then left overnight then washed off by the participant or parent with plain warm water in the morning. There was no second treatment.

For the other group, we also applied the product to dry hair and dried off excess water with the

171 hair dryer to the same sticky stage. This was then left for a timed 2 hour period before being rinsed 172 off with plain warm water by the investigator. A repeat treatment was given 7 days later. 
174 For both groups, participants were given bottles of the same non-medicated shampoo to use for

175 normal hair washing on the third and tenth days after treatment.

176

177 Participants

178 Participants were recruited from respondents to an invitation letter and information sheet

179 distributed through schools or via general practitioners. Parents of children with lice telephoned

180 the study co-ordinator and made an appointment for a home visit. We visited within 24 hours to

181 check prospective participants for living lice using a plastic detection comb (Albyn of Stonehaven,

182 Stonehaven, Scotland). If an infestation was found, consisting of one or more live lice, the person

183 was invited to join the study and the parent of guardian guided through a written consent

184 procedure followed by child assent. All household members were offered examination and, if

185 found to be infested, the opportunity to join the study if fitting the enrolment criteria. In this

186 context the intensity of an infestation was partially subjective: Heavy = more than one louse found

187 with the first stroke of the comb; Medium = one louse found with the first stroke; and Light = lice

188 found only after several strokes of the comb over different parts of the head. Lice were returned to

189 the head because treatment followed. The number of participants with each level of infestation is

190 shown in Tables 1 and 2.

191

192 Consenting participants provided baseline demographic data including age, gender, hair

193 characteristics, concurrent medications, and medical history. Some demographic characteristics,

194 such as hair dryness and thickness, were subjective assessments made by the investigators on the

195 day and were intended to serve only as a guideline. In both studies the lower age limit was 4

196 years. People who were sensitive to paraben preservatives; who had persistent disorders of the

197 scalp such as eczema or psoriasis; who had received a head louse treatment within the 4 weeks

198 prior to entry; or had undergone antibiotic treatment or had their hair bleached, colour treated, or

199 permanently waved within the previous 4 weeks, were excluded. All treatments and assessments

200 were performed in the participant's home. No payment was offered for participation.

201

202 For the first study, between July 1998 and January 1999, we recruited 44 participants (35 children

203 and 9 adults), out of the 120 planned, divided equally between cocamide DEA $10 \%$ and 1\%

204 permethrin creme rinse treatments (Figure 1). Of these one person treated with permethrin was 
205 lost to follow up before any post-treatment checks could be made. Six others (four treated with 206 cocamide DEA and two with permethrin) were withdrawn at the participant's request due to lack

207 of efficacy. This left 19 participants treated with cocamide DEA and 18 with permethrin with

208 complete data sets before the sponsor requested an early termination on grounds of lack of

209 efficacy for both products.

210

211 In the second study we recruited 112 out of 120 planned enrolments between October and

212 December 1999. All participants were treated with cocamide DEA $10 \%$ divided between two

213 treatment regimens: either a single overnight application or two 2 hour applications a week apart.

214 There were seven participants withdrawn (Figure 2). Three people, one from the single

215 application group and two from the double application group dropped out because a baby from the

216 family was hospitalised and the others were unable to keep appointments. Four participants were

217 withdrawn by investigators due non-compliance, one from the single application group and three

218 from the double application group. This left 54 people treated with one overnight application and

21951 who received two 2 hour applications. Recruitment for this study was also terminated early at

220 the sponsor's request on grounds of lack of efficacy.

221

222 Ethics

223 Ethical approval was granted by the Huntingdon Local Research Ethics Committee of

224 Cambridgeshire Health Authority for the first study (protocol CTRL01, REC reference 97/229, 225 dated $25^{\text {th }}$ July 1997). The second study received approval from both Huntingdon LREC and

226 Cambridge Local Research Ethics Committee (protocol CTRL02, REC reference 99/211, dated

227 17th September 1999). A Clinical Trial Exemption Certificate (CTX16442/0001/A) was granted

228 by the UK Medicines Control Agency to permit conduct of each of the trials.

229

230 In each study, parents/guardians stated that they understood the purpose of the study and they

231 agreed to abide by the requirements of the protocol before providing witnessed written consent for

232 participating children under the age of 16 years.

233

234 The studies were conducted in conformity with the principles of the Declaration of Helsinki and

235 the ICH Guidelines for Good Clinical Practice (GCP) prevailing at the time. 
237 These studies were not registered on a publically accessible database at the time they were 238 conducted because there was no facility at the time for doing so. Both studies have now been 239 retrospectively registered on the database at https://clinicaltrials.gov with the registration number 240 NCT02500524 for the cocamide DEA versus permethrin study (CTRL01) and NCT02499549 for

241 the comparison between treatment regimens using cocamide DEA (CTRL02).

242

243

\section{Outcome measures}

244 For both studies the primary outcome measure was elimination of infestation following completion of the allocated treatment regimen. We used a plastic louse detection comb on dry hair for follow up examinations looking for lice. In the first study assessments were made on Days 4, 7, 10 and 14 after treatment. A similar approach was taken for the second study but, because one arm of the study used a two application regimen, the timing was adjusted so that checkups were made on Days 4, 8, 11, and 14 after the first application of treatment. Any live lice found during these visits were collected on the case record forms.

Sample size

253 Both studies were designed to demonstrate equivalence to within $20 \%$ between treatments.

254 Sample sizes were estimated on the basis that it would be possible to detect equivalence with $95 \%$ 255 confidence, assuming that the underlying rates of efficacy would be $90 \%$. At this level we estimated that a group size of 50 would provide $80 \%$ power and 61 would give $90 \%$ power. Therefore, a sample size of 60 per group was selected on the basis that it would provide at least $85 \%$ power, even allowing for possible post-randomisation protocol violations. The estimate also showed that if the underlying rates of the treatments averaged $90 \%$, this sample size would also have a power of $90 \%$ to detect a difference of around $18 \%$ with $95 \%$ confidence.

\section{Randomisation and allocation concealment}

263 Randomisation codes for both studies were produced from computer generated lists by an

264 independent statistician appointed by the sponsor. Allocation sequences were made in balanced

265 blocks of 12 and the identification of each treatment entered on instruction sheets enclosed in 266 sealed, numbered, opaque envelopes. The study numbers were allocated in sequence and the 
267 identity of the allocated treatment only revealed after receipt of informed consent and

268 confirmation of suitability to participate in the study. Post-treatment assessments were blinded

269 and performed by different investigators unaware of the allocated treatment.

270

271 Statistical methods

272 The outcomes of tests conducted in vitro were analysed using a purpose built calculator for

273 estimating the $95 \%$ confidence interval (CI) from the normal approximation to the binomial

274 distribution. Overall, with the high level of treatment failure in each of the treatments arms in

275 both clinical studies, combined with the sponsor request to terminate both studies early, the

276 intended analyses could not be performed in the way intended. However, we were able to

277 analyse differences between the groups in terms of efficacy, safety, ease of use, and acceptability

278 using the intention to treat population for both studies by Fisher's exact test and unstratified chi-

279 squared tests for yes/no variables and the Kruskal-Wallis test for ranked variables. The initial

280 analyses were expected to be performed by the consultant statistician (PN Lee Statistics and

281 Computing Ltd, Sutton, UK) using bespoke software but because the studies were terminated

282 early the sponsor decided not to proceed with this work. We also planned to test for equivalence

283 using the per-protocol population based on $95 \%$ confidence limits derived from the normal

284 approximation. However, because both studies were terminated early a true per-protocol group

285 was not determined.

286

287

288

\section{Results}

Pre-clinical studies

289

In the first series of in vitro tests, comparing the $3.5 \%$ and $10 \%$ lotions that we performed prior to clinical studies, we found the $10 \%$ mixture was the more effective using an exposure for 60 minutes followed by washing with shampoo that resulted in the death of all but one laboratory reared clothing/body lice when reviewed the following day (Table 1). The effect on louse eggs was also encouraging indicating that a high proportion of eggs could be inhibited from hatching, with a significant ( $\mathrm{p}<0.01,95 \%$ CI 0.014 to 0.085 ) increase in the proportion of eggs that showed no sign of development post-treatment compared with the control group, suggesting penetration of the egg structure by the active component of the lotion (Table 2). 
298 Because it was clear from the first clinical study that inability to kill the louse eggs contributed

299 significantly to the failure to cure we conducted a second in vitro study to investigate whether

300 older eggs were harder to kill. In the original in vitro test louse eggs 24-48 hours old were used

301 whereas on the head there would be eggs at different development stages from newly laid through

302 to hatching. We found that as the eggs aged they became less susceptible to the treatment, with 5

303 day and older eggs requiring exposures longer than 2 hours to stop louse nymphs from emerging

304 and by the seventh day not all eggs were prevented from hatching using an overnight exposure. In

305 addition the proportion of embryos that failed to develop eyespots (the first definitive indication of

306 embryonic development) was lower when exposed to a 2 hour treatment than overnight and by the

$3076^{\text {th }}$ day there was no difference from the control group because all embryos that would develop had

308 reached the "eyespot" stage between days 5 and 6 (Table 3).

309

310 Increasing the dose concentration of cocamide DEA, by prolonging the treatment time or speeding

311 up the evaporation rate of the excipient water using a hair dryer, produced some increase in

312 activity, with no significant increase in overall inhibition of hatching but with a significant $(\mathrm{p}<$

$3130.001,95 \%$ CI 0.131 to 0.266 ) increase in the proportion of eggs failing to develop after hair

314 drying, whereas inhibiting water evaporation in a saturated atmosphere appeared to reduce the

315 activity (Table 4). Use of shampoo to remove the product rather than simple water rinsing was

316 potentially the most important factor for reduction of activity ( $p=0.0004,95 \%$ CI 0.078 to 0.304 ),

317 especially against older louse eggs, which showed no significant difference from the control group

318 on overall failure to hatch (Table 4).

319

320 Clinical studies

321 Participant flow

322 Over the two studies, we recruited participants from 82 households ranging in size from 2 to 8

323 members (mean 4.56), with between 1 and 5 people (mean 1.93) enrolled. In both studies the

324 majority of households had either four or five members. In the first study, comparing cocamide

325 DEA with permethrin, there were no significant demographic differences between the groups

326 (Table 5). A similar demographic was observed in the second study, comparing the two treatment

327 regimens of cocamide DEA, with the exception of a significant $(\mathrm{p}<0.038)$ difference in 
328 proportion of 10-14 year old participants (Table 6) and a non-significant trend $(\mathrm{p}=0.073)$ in the

329 single application group for more people to have "Average" thickness hair.

330

331 Outcomes

332 In the comparison between a single 1 hour application of cocamide DEA 10\% lotion and one 10

333 minute application of $1 \%$ permethrin creme rinse, we found no significant difference between the

334 treatments. Both treatments performed badly in terms of efficacy with success in $3 / 23(13.0 \%)$ of

335 participants treated with cocamide DEA ( 1 cure, and 2 cases of reinfestation after cure), and 5/21

$336(23.8 \%)$ cures, and no cases of reinfestation, in the permethrin group. This difference was not

337 significant $(\mathrm{p}=0.355)$ (OR $0.48,95 \%$ CI 0.099 to 2.319$)$. The relative severity of failure in most

338 participants was such that four participants were withdrawn from the cocamide DEA group and

339 two from the permethrin group in order to minimise the continuing irritation from infestation. In

340 addition one person in the permethrin group was lost to follow up without being assessed for

341 efficacy.

342

343 In the second study the single overnight application achieved 10/56 (17.9\%) successful treatments

344 (8 cured, and 2 reinfested) compared with 19/56 (33.9\%) success (16 cured and 2 reinfested) for

345 the 2 hour application regimen repeated after 1 week. This difference was also not significant $(p=$

3460.0523 ) (OR $0.423,95 \%$ CI 0.176 to 1.020$)$. One person withdrew from the single treatment

347 regimen immediately after treatment because it was uncomfortable and in the double treatment

348 group there was a drop out and a case of non-compliance in which non-study treatments were

349 used.

350

351 Since the outcomes of the second study showed a poor level of efficacy for the cocamide DEA

352 lotion, even with longer application times that in vitro tests had indicated would be more effective,

353 we decided to run a comparison in vitro of the ovicidal effect of the two batches of product. In

354 this series of tests we found that the retained samples of the earlier batch of product (Batch

355 number LI 35101) were more effective than the batch used in the second study (Batch number NH

356 35101) (Table 7). The differences were highly significant $(\mathrm{p}<0.00001)$ for both a 2 hour

357 treatment and an overnight treatment suggesting that if we had used the earlier material in the

358 second trial it could have proved more effective. 
360 Most users of the cocamide DEA 10\% lotion reported seeing large numbers of dead lice on their

361 pillow the morning after treatment and some reported seeing darkly coloured dead lice washed

362 from the hair as the product was rinsed out. This was particularly reported by parents who chose

363 to wash their children's hair in the bath with the result that the dead insects were seen floating on

364 the surface of the bath water.

365

366

367

In the first study, this was the first experience of head louse infestation for two, and in the second study for five, of the participants. All other participants claimed to have been treated

368

369

370

371

372

373

374

375

376

377

378

379

380

381

382

383

384

385

386

387

388

389

unsuccessfully prior to entry into the study using one or more insecticide or essential oil based

products, in most cases alternating with some kind of combing process, including six people from the first study and 27 from the second who previously had used only wet combing with conditioner. Apart from wet combing with conditioner, the majority of treatments used also had a conditioner-type base (cetyl alcohol emulsion) so the previous treatments had all left residues of conditioning lipids on the hair, which were completely removed by the cocamide DEA. As a result all participants/parents except one reported that the treated hair recovered its normal lustre and texture, which had been obscured by the conditioning chemicals, because the product had stripped away all the excess lipid from the hair shafts.

In the second study bottles of product were weighed before and after use to determine how product much was used during each treatment. This ranged from 23.5 grams up to 260.3 grams (mean 94.0 grams) during a single application, depending upon the length and thickness of the hair.

The raw data for both studies as extracted from the case records are appended as Supplemental Information files.

\section{Adverse events}

Across the two studies there were 20 reported adverse events. None of these were serious and; apart from two that were attributed to viral infections ( 1 head cold, 1 sore throat with fever), one of continued itch due to lice from before treatment, and one in which a child was sprayed in the face with an unrelated detergent product by a sibling; all events were some form of application site 
390 reaction to the treatment. All these reactions were of short duration and all resolved spontaneously

391 (Table 8).

392

393 Discussion

394 Cocamide diethanolamine (cocamide DEA) is a powerful surfactant and foaming agent formerly 395 quite widely used in toiletry shampoos. Currently its use in this context is mostly limited to so-

396 called clarifying or stripping shampoos designed to remove lipid and other chemical deposits from

397 hair so that further treatments for permanent waves or colouring can be applied and until recently

398 was considered safe in leave on preparations containing 10\% of the active substance (Cosmetic

399 Ingredient Review Expert Panel , 1996). However, since this study was conducted the compound

400 has been reclassified by the International Agency for Research on Cancer (a World Health

401 Organization body) in Group 2B, compounds possibly carcinogenic to humans (International

402 Agency for Research on Cancer, 2013).

403

404 It can be assumed that any activity of cocamide DEA against head lice originates in the surfactant

405 activity of the compound to emulsify some of the lipid protective waterproofing layer of the

406 cuticle of the insects. The result would be that, as the material is rinsed off, the emulsified lipids

407 would be taken with it leaving the insects susceptible to dehydration because the protective layer

408 on the surface of the cuticle is damaged. Observations of treated lice confirmed that conclusion,

409 initially showing a knockdown-like reaction in which the limbs contracted, and then appearing

410 shrunken and dehydrated when washed from the hair.

411

412 Our studies showed that cocamide DEA 10\% was able to kill many lice but was ineffective against 413 a proportion of the insects. With the shorter application time of 60 minutes (study 1) there would

414 have been little concentration effect due to evaporation and with the relatively runny lotion it was

415 possible that not all lice were adequately coated with the fluid and could have survived as a result.

416 However, in the second study both treatment regimens involved deliberate concentration of the

417 lotion on the hair using a hand held dryer, which ensured that all parts of the hair and scalp were

418 coated with the viscous residue. Nevertheless some lice and louse eggs survived the exposure on

419 most participants.

420 
421 The low level of activity in our studies contrasts starkly with the claims of $100 \%$ efficacy with a 422 single 60 minute application made in the report of a previous uncontrolled study (Mülhofer, 423 1994). In that study the participants were vigorously brushed, with a bristle brush, so that lice 424 undergoing a knockdown effect were dislodged from the scalp and removed by the investigators. 425 However, when the participants were followed up it is puzzling that no louse eggs were reported 426 to have hatched subsequent to treatment, especially since each of the participants was treated using 427 only $5-10 \mathrm{ml}$ of the product. In contrast we used over $90 \mathrm{ml}$ for an average treatment in our second 428 study and the treatments were applied for at least double the time of 1 hour used in the earlier trial 429 (Mülhofer, 1994), which suggests either that cocamide DEA had become significantly less 430 effective against European lice in fewer than 5 years, and in an area where it had not previously 431 been used, or else there was something seriously wrong with the assessment and follow up 432 methodology used in the Austrian study.

434 The stark contrast in effect observed in our post-trial comparison of the two batches of lotion using 435 in vitro testing suggests that there was considerable variability in the active materials used. Both 436 batches were investigational preparations made up in the laboratory of the sponsor, using different 437 batches of raw material. Why such a difference was observed in tests against the same group of 438 louse eggs could not be explained other than as a result of differences in the bioavailability, and 439 activity, of the active material in the two batches of formulation.

441 The use of surfactants for control of louse infestations is not novel, with folk-lore remedies dating 442 back centuries using materials like saponins as part of treatment. But in the modern era no 443 treatment had employed detergents or surfactants alone for killing the insects until quite recently. 444 However, there are a number of reports of effectiveness of surfactant based materials from the 445 patent literature such as non-vicinal diols (Lover, Singer \& Rhodes, 1981c); aliphatic or aryl 446 aliphatic alcohols and aliphatic esters (Stafford Miller, 1979); substituted monohydric alcohols 447 (Lover et al. 1981d); higher alcohols (Stafford Miller, 1982); glycine derivatives (Lover et al., 448 1981b); polyoxethylene derivatives (Lover et al., 1981a); imidazoline (Miranol ${ }^{\mathrm{TM}}$ ) surfactant 449 compounds (Lover, Singer \& Lynch, 1980); aminoproprionic acid derivative surfactants (Lover et 450 al., 1978), although none of these materials was ever used commercially. Since then one shampoo 451 based on cocamide DEA and other coconut derived surfactants has been reported to show efficacy 
452 (Connolly et al., 2009). More recently there have been investigations of vicinal diols, a group of 453 compounds little used in any application before the 1990s and which are also derived from 454 coconut, some of which were found to be effective against lice (Campbell \& Carver, 2002). One 455 of these, 1,2-octanediol, has also been clinically tested and shown to be effective when 456 incorporated into the right vehicle using a 5\% concentration (Burgess et al., 2012). All of these 457 are believed to act on the surface lipids of the louse cuticle to disrupt the waterproofing layer so 458 that the insects lose water uncontrollably and dehydrate (Burgess et al., 2012). From this is can be concluded that surfactants can be effective to kill and eliminate head lice. However, simply making a solution of a powerful surfactant like cocamide DEA, even when subsequently

461 462 463 464 465 466

467 468

concentrated by evaporation of the water from the mixture, does not necessarily provide adequate activity to disrupt the cuticle lipid on all lice and demonstrates the importance of proper formulation as a major factor in achieving sufficient activity.

\section{Acknowledgements}

We would like to thank Dr Angela Owen-Smith, former consultant paediatrician, who acted as local medical contact for the first study, and Dr Nick Irish, former consultant in communicable disease control, who was medical contact for the second study. Peter N Lee provided statistical advice during the design stages of the first study. Investigation team members who contributed to the studies, but who are not named as authors, were Nazma A Burgess, Rachel Cooper, Ann Scarlett, and David Thomson.

\section{References}

Burgess I. 1991. Malathion lotions for head lice - a less reliable treatment than commonly believed. Pharmaceutical Journal 247: 630-632.

Burgess IF, Lee PN, Kay K, Jones R, Brunton ER. 2012. 1,2-octanediol, a novel surfactant, for treating head louse infestation: identification of activity, formulation, and randomised, controlled trials. PLoS ONE 7(4): e35419. DOI: 10.1371/journal.pone.0035419. 
482 Campbell J, Carver A. 2002. Pesticides based on vicinal diols. International Patent; WO 483 02/069707 Al.

484

485 Connolly M, Stafford KA, Coles GC, Kennedy CT, Downs AM. 2009. Control of head lice with 486 a coconut-derived emulsion shampoo. Journal of the European Academy of Dermatology and 487 Venereology 23: 67-69. DOI: 10.1111/j.1468-3083.2008.02829.x 488 489 Cosmetic Ingredient Review Expert Panel. 1996. Amended final report of the safety assessment 490 of cocamide DEA. Journal of the American College of Toxicology 15: 527-542.

491 DOI: $10.3109 / 10915819609008729$

492

493

Heukelbach J, Speare R, Canyon D. 2009. Natural products and their application to the control of 494 head lice: an evidence-based review. In: Brahmachari G, ed. Chemistry of Natural Products: 495 Recent Trends \& Developments. Trivandrum, Kerala: Research Signpost, 277-302.

496

497

498

International Agency for Research on Cancer. 2013. Coconut oil diethanolamine condensate. IARC Monographs 101: 141-148.

499

500

Lover MJ, Singer AM, Lynch DM. 1980. Ectoparasite toxicants containing imidazoline. US

501 Patent, 4,238,499.

502

503

Lover MJ, Singer AM, Lynch DM, Rhodes WE $3^{\text {rd }}$. 1978. Aminoproprionic-acids as

504 ectoparasiticides. US Patent, 4,126,700.

505

506

Lover MJ, Singer AM, Lynch DM, Rhodes WE $3^{\text {rd }}$. 1981a. Use of polyoxethylene derivatives as 507 ectoparasiticides. UK Patent, GB1 604622.

508

509 Lover MJ, Singer AM, Lynch DM, Rhodes WE $3^{\text {rd }}$. 1981b. Use of glycine derivatives as ovicides 510 or insecticides. UK Patent, GB1 604854. 
512 Lover MJ, Singer AM, Rhodes WE 3 ${ }^{\text {rd }}$, Bilodeau WN. 1981c. Use of certain polyol toxicants as

513 ectoparasiticides or ovicides. UK Patent, GB1 604856.

514

515 Lover MJ, Singer AM, Lynch DM, Rhodes WE $3^{\text {rd }}$, Bilodeau WN. 1981d. Ectoparasiticidal

516 toxicants. UK Patent, GB1 604859.

517

518 Mülhofer AJ. 1994. Pediculus humanus in man. Comparative study of the action of the

519 biological product Läuse Schocker für Menschen against head lice in humans, acute and sub-

520 acute. LHS Institut für Hygieneforschung und Schädlingsbekämpfung in Labor und Praxis.

521

522 Stafford Miller Ltd. 1979. Pediculicidal toxicants. UK Patent, GB1 547020.

523

524 Stafford Miller Ltd. 1982. Use of higher alcohols as toxicants against lice. UK Patent, GB1 604 525857.

526

527

Takano-Lee M, Edman JD, Mullens BA, Clark JM. 2004. Home remedies to control head lice:

528

Assessment of home remedies to control the human head louse, Pediculus humanus capitis

529

(Anoplura: Pediculidae). Journal of Pediatric Nursing 19: 393-398.

530

DOI: http://dx.doi.org/10.1016/j.pedn.2004.11.002

531

532 Veal L. 1996. The potential effectiveness of essential oils as a treatment for headlice, Pediculus

533 humanus capitis. Complementary Therapies in Nursing and Midwifery 2: 97-101.

534

535 Yang YC, Lee HS, Clark JM, Ahn YJ. 2004. Insecticidal activity of plant essential oils

536 against Pediculus humanus capitis (Anoplura: Pediculidae). Journal of Medical Entomology 41:

537 699-704.

538

539

540

541

542

543

544

List of Figures 
Figure 1. Flowchart of participants through the first clinical study

549

Figure 2. Flowchart of participants through the second clinical study

550

551

552 


\section{Figure 1 (on next page)}

Flowchart of participants through the first clinical study 


\section{Assessed for eligibility}

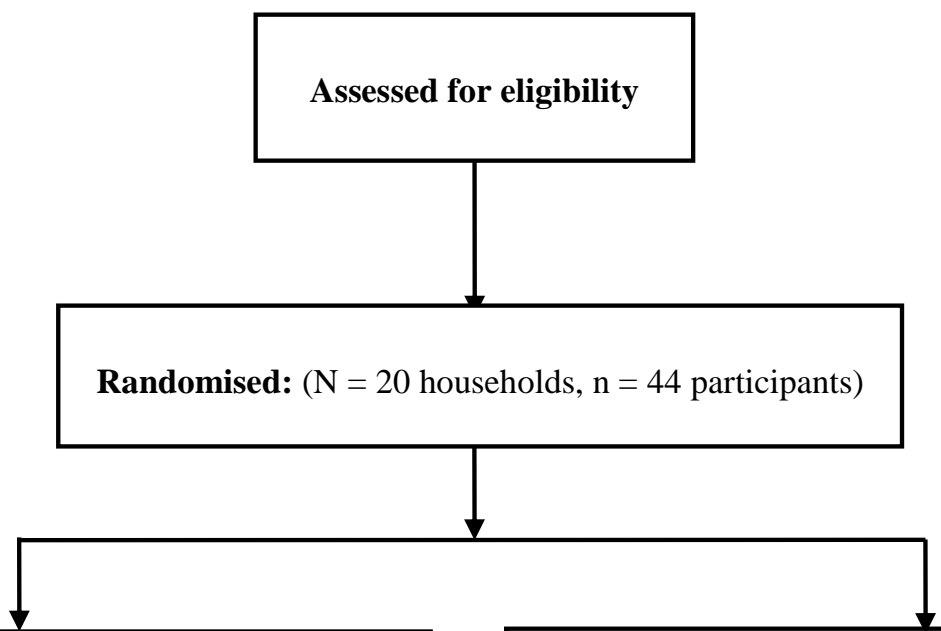

Allocated to receive $10 \%$ cocamide DEA lotion ( $\mathrm{n}=23$ participants)

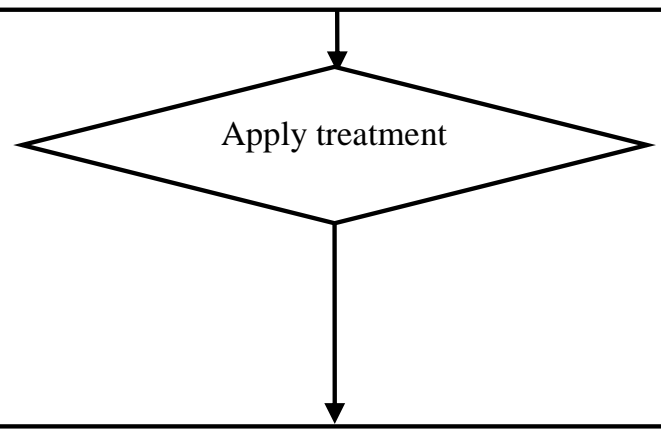

Monitor adverse experiences

Follow up check by detection combing Day 4: if lice present - collect on CRF

Day 7: if lice present - collect on CRF

Day 10: if lice present - collect on CRF

Day 14: if lice present - collect on CRF
Allocated to receive $1 \%$ permethrin creme rinse ( $\mathrm{n}=21$ participants $)$

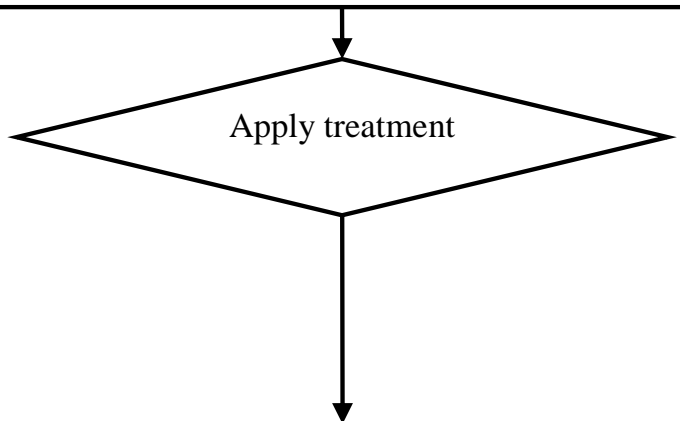

Monitor adverse experiences

Follow up check by detection combing Day 4: if lice present - collect on CRF Day 7: if lice present - collect on CRF Day 10: if lice present - collect on CRF Day 14: if lice present - collect on CRF

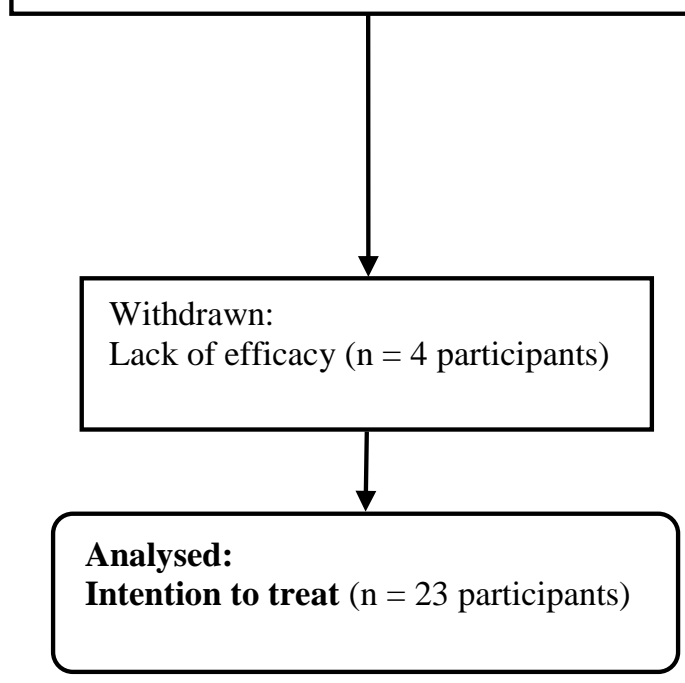

Lost to follow up ( $\mathrm{n}=1$ participant $)$ Withdrawn:

Lack of efficacy ( $\mathrm{n}=2$ participants)

Analysed:

Intention to treat ( $\mathrm{n}=21$ participants) 
Figure 2 (on next page)

Flowchart of participants through the second clinical study 


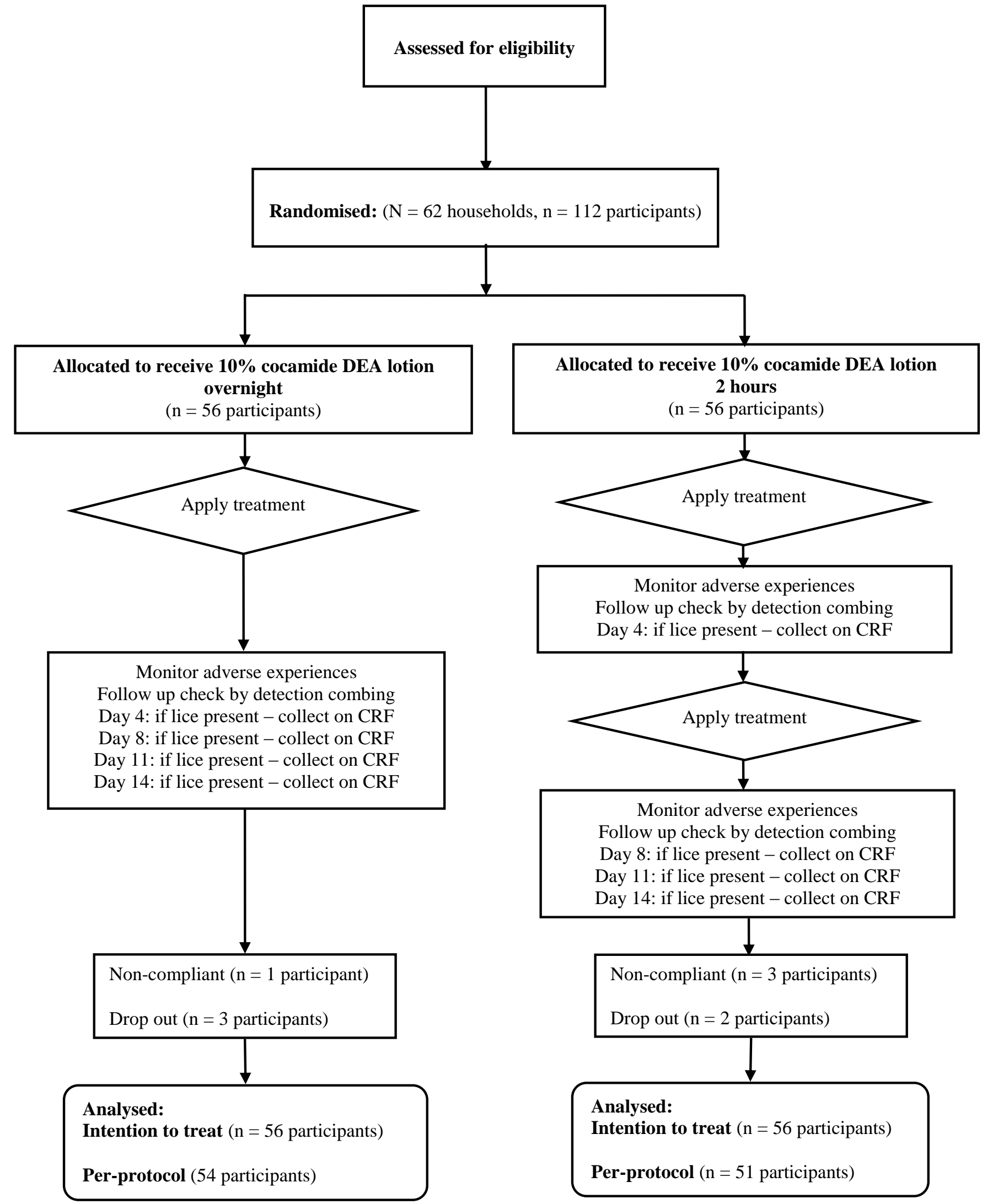




\section{Table 1 (on next page)}

Activity of two concentrations of cocamide DEA lotion against lice in vitro 
1

2

3

\begin{tabular}{|c|c|c|c|c|c|}
\hline \multirow[t]{2}{*}{ Treatment } & \multicolumn{4}{|c|}{ Number of lice from three replicates of 20} & \multirow[b]{2}{*}{ Mortality \% } \\
\hline & Total & Killed & Immobile & Mobile & \\
\hline Cocamide DEA 3.5\% & 62 & 51 & 6 & 5 & 91.9 \\
\hline Cocamide DEA $10 \%$ & 62 & 54 & 7 & 1 & 98.4 \\
\hline Water control & 62 & 5 & 4 & 53 & 14.5 \\
\hline & & & & & \\
\hline
\end{tabular}

4 


\section{Table 2 (on next page)}

Activity of $10 \%$ cocamide DEA lotion against freshly laid louse eggs in vitro 
2

3

\begin{tabular}{|l|c|c|c|c|c|c|}
\hline Treatment & \multicolumn{3}{|c|}{ Number of eggs from three replicate tests } & Mortality \% \\
\hline & Total & Hatched & $\begin{array}{c}\text { Half- } \\
\text { hatched }\end{array}$ & Died & Undeveloped & \\
\hline & & & & & & \\
\hline Cocamide DEA 10\% & 569 & 3 & 1 & 495 & 70 & 99.3 \\
\hline Water control & & & & & & \\
\hline & 518 & 458 & 3 & 19 & & \\
\hline
\end{tabular}

4 


\section{Table 3(on next page)}

Effect of different application times using 10\% cocamide DEA lotion on louse eggs at different development ages 


\begin{tabular}{|c|c|c|c|c|c|c|}
\hline \multirow{2}{*}{$\begin{array}{l}\text { Age of eggs - } \\
\text { application } \\
\text { time }\end{array}$} & \multicolumn{5}{|c|}{ Number of eggs from three replicate tests } & \multirow[t]{2}{*}{$\begin{array}{c}\text { Mortality \% } \\
\text { (Undeveloped \%) }\end{array}$} \\
\hline & Total & Hatched & $\begin{array}{c}\text { Half- } \\
\text { hatched }\end{array}$ & Died & Undeveloped & \\
\hline \multicolumn{7}{|l|}{ Cocamide DEA } \\
\hline $\begin{array}{l}1 \text { day old }-2 \\
\text { hours }\end{array}$ & 353 & 0 & 0 & 56 & 297 & $\begin{array}{c}100 \\
(84.1)\end{array}$ \\
\hline $\begin{array}{l}1 \text { day old - } \\
\text { overnight }\end{array}$ & 291 & 0 & 0 & 3 & 288 & $\begin{array}{c}100 \\
(99.0)\end{array}$ \\
\hline $\begin{array}{l}4 \text { days old }-2 \\
\text { hours }\end{array}$ & 278 & 15 & 20 & 189 & 54 & $\begin{array}{c}94.6 \\
(19.4)\end{array}$ \\
\hline $\begin{array}{l}4 \text { days old - } \\
\text { overnight }\end{array}$ & 283 & 0 & 13 & 43 & 227 & $\begin{array}{c}100 \\
(80.2)\end{array}$ \\
\hline $\begin{array}{l}5 \text { days old }-2 \\
\text { hours }\end{array}$ & 278 & 0 & 38 & 71 & 169 & $\begin{array}{c}100 \\
(60.8)\end{array}$ \\
\hline $\begin{array}{l}5 \text { days old - } \\
\text { overnight }\end{array}$ & 300 & 0 & 0 & 218 & 82 & $\begin{array}{c}100 \\
(30.2)\end{array}$ \\
\hline $\begin{array}{l}6 \text { days old }-2 \\
\text { hours }\end{array}$ & 271 & 84 & 90 & 82 & 14 & $\begin{array}{l}69.0 \\
(5.2)\end{array}$ \\
\hline $\begin{array}{l}6 \text { days old - } \\
\text { overnight }\end{array}$ & 324 & 0 & 1 & 293 & 30 & $\begin{array}{l}100 \\
(9.3)\end{array}$ \\
\hline $\begin{array}{l}7 \text { days old - } \\
\text { overnight }\end{array}$ & 231 & 47 & 42 & 121 & 21 & $\begin{array}{l}79.7 \\
(9.1)\end{array}$ \\
\hline $\begin{array}{l}8 \text { days old - } \\
\text { overnight }\end{array}$ & 137 & 28 & 2 & 101 & 6 & $\begin{array}{l}79.6 \\
(4.4)\end{array}$ \\
\hline Water control & 328 & 310 & 4 & 4 & 10 & $\begin{array}{c}5.5 \\
(3.1)\end{array}$ \\
\hline
\end{tabular}


Table 4 (on next page)

Effect of different washing or incubation regimens on effect of cocamide DEA lotion on louse eggs 


\begin{tabular}{|c|c|c|c|c|c|c|}
\hline \multirow{2}{*}{ Treatments } & \multicolumn{5}{|c|}{ Number of eggs from three replicate tests } & \multirow{2}{*}{$\begin{array}{c}\text { Mortality \% } \\
\text { (Undeveloped \%) }\end{array}$} \\
\hline & Total & Hatched & $\begin{array}{c}\text { Half- } \\
\text { hatched }\end{array}$ & Died & Undeveloped & \\
\hline \multicolumn{7}{|l|}{ Cocamide DEA } \\
\hline & & & & & & \\
\hline \multicolumn{7}{|l|}{1 day old eggs } \\
\hline $\begin{array}{l}60 \text { minutes - } \\
\text { shampoo wash }\end{array}$ & 117 & 80 & 1 & 30 & 6 & $\begin{array}{l}31.6 \\
(5.1) \\
\end{array}$ \\
\hline $\begin{array}{l}60 \text { minutes - } \\
\text { water rinse }\end{array}$ & 205 & 101 & 5 & 92 & 7 & $\begin{array}{l}50.7 \\
(3.4) \\
\end{array}$ \\
\hline $\begin{array}{l}120 \text { minutes - } \\
\text { water rinse }\end{array}$ & 111 & 7 & 1 & 94 & 9 & $\begin{array}{l}93.7 \\
(8.1) \\
\end{array}$ \\
\hline $\begin{array}{l}60 \text { minutes - } \\
\text { humidified + } \\
\text { water rinse }\end{array}$ & 247 & 139 & 6 & 95 & 7 & $\begin{array}{l}43.7 \\
(2.8)\end{array}$ \\
\hline $\begin{array}{l}60 \text { minutes }+ \\
\text { drying - water } \\
\text { rinse }\end{array}$ & 159 & 64 & 0 & 58 & 37 & $\begin{array}{c}59.8 \\
(23.3)\end{array}$ \\
\hline \multicolumn{7}{|l|}{5 day old eggs } \\
\hline & & & & & & \\
\hline $\begin{array}{l}60 \text { minutes - } \\
\text { water rinse }\end{array}$ & 117 & 100 & 7 & 3 & 7 & $\begin{array}{l}14.5 \\
(6.0) \\
\end{array}$ \\
\hline Water control & 203 & 187 & 2 & 6 & 8 & $\begin{array}{c}7.9 \\
(3.9)\end{array}$ \\
\hline
\end{tabular}




\section{Table 5 (on next page)}

Disposition of demographic characteristics of participants in the first clinical study 
1

2

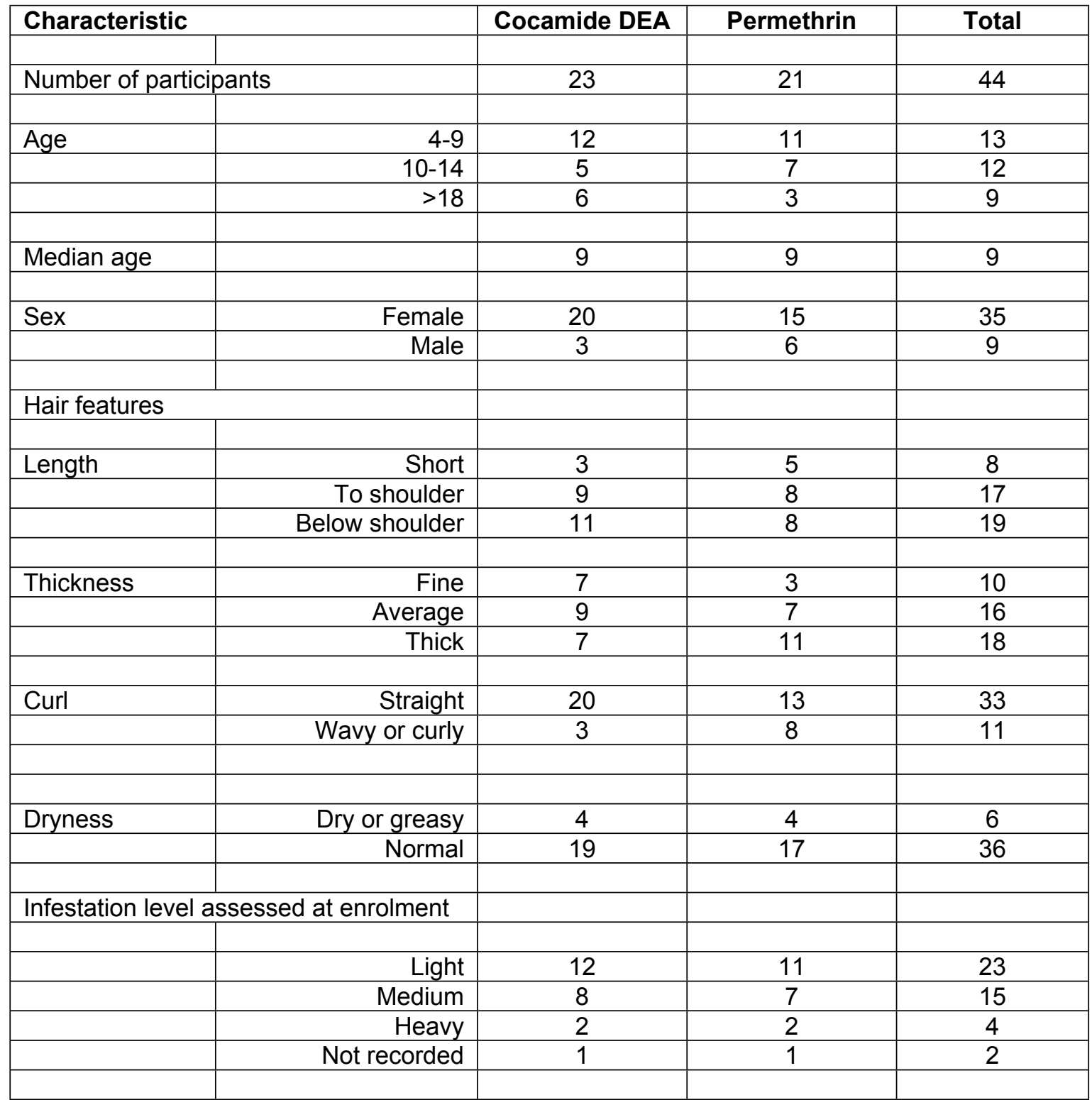

3 
Table 6(on next page)

Disposition of demographic characteristics of participants in the second clinical study 
2

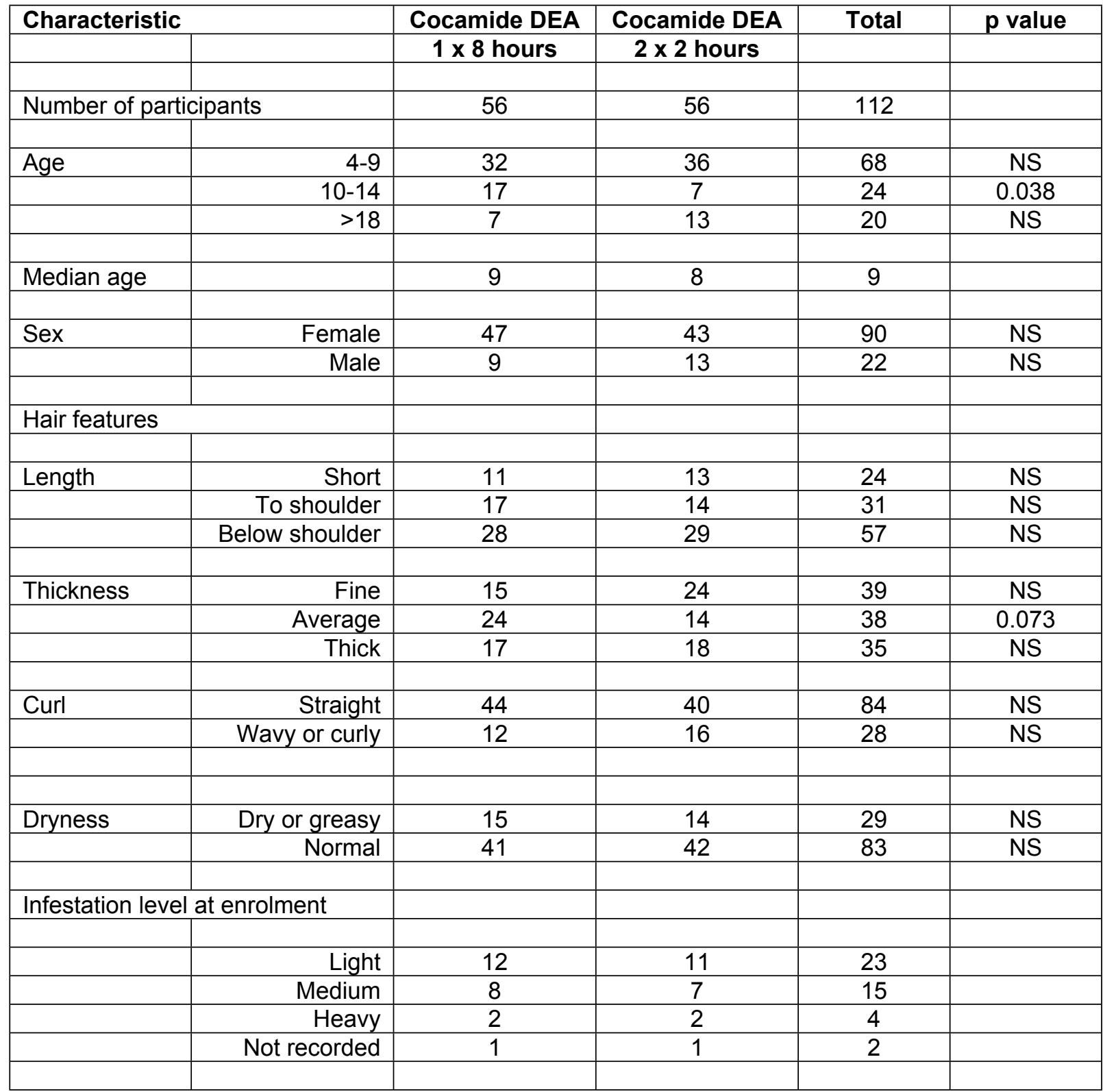




\section{Table 7 (on next page)}

Comparison of the two product batches used in the clinical studies for ovicidal activity in vitro 


\begin{tabular}{|c|c|c|c|c|c|c|}
\hline \multirow{2}{*}{ Treatment } & \multirow{2}{*}{ Time } & \multicolumn{4}{|c|}{ Number of eggs } & \multirow{2}{*}{$\begin{array}{c}\text { Mortality\% } \\
\text { (Undeveloped \%) }\end{array}$} \\
\hline & & Total & Hatched & Died & Undeveloped & \\
\hline $\mathrm{NH} 35101$ & 2 hours & 411 & 357 & 24 & 30 & $13.1 \%(7.3 \%)$ \\
\hline $\mathrm{NH} 35101$ & Overnight & 291 & 100 & 37 & 154 & $65.6 \%(52.9 \%)$ \\
\hline LI 35101 & 2 hours & 391 & 39 & 318 & 34 & $90.0 \%(8.7 \%)$ \\
\hline LI 35101 & Overnight & 389 & 0 & 3 & 386 & $100 \%(99.2 \%)$ \\
\hline Control & & 591 & 533 & 16 & 42 & $9.8 \%(7.1 \%)$ \\
\hline
\end{tabular}

3 


\section{Table 8 (on next page)}

Adverse events possibly or probably related to treatment in the two clinical studies 


\begin{tabular}{|c|c|c|c|}
\hline Participant & Treatment & Adverse event & Duration of event \\
\hline \multicolumn{4}{|l|}{ Study 1} \\
\hline 002 & Permethrin 10min & Itch when washed off & 5 minutes \\
\hline & & & \\
\hline 009 & C-DEA 60min & Itch when washed off & 5 minutes \\
\hline 011 & C-DEA 60min & Itch when washed off & 5 minutes \\
\hline 018 & C-DEA 60min & Itch during and after treatment & 60 minutes \\
\hline 019 & C-DEA 60min & Itch during and after treatment & 60 minutes \\
\hline \multicolumn{4}{|l|}{ Study 2} \\
\hline & & & \\
\hline 004 & C-DEA $1 \times \mathrm{O} / \mathrm{N}$ & Itch after treatment & 2-3 days \\
\hline 007 & C-DEA 1x O/N & Itch from before treatment & 4 days \\
\hline 008 & C-DEA $1 \times \mathrm{O} / \mathrm{N}$ & Stinging eyes & During treatment \\
\hline 033 & C-DEA 1x O/N & Rash on neck & 4-5 days \\
\hline 070 & C-DEA 1x O/N & Wheals/rash on chest $24 \mathrm{~h}$ after treatment & 2-3 days \\
\hline 097 & C-DEA $1 \times$ O/N & Intermittent itch & 2-3 days \\
\hline 100 & C-DEA 1x O/N & Intermittent itch & 2-3 days \\
\hline 014 & C-DEA $2 \times 2 h$ & Itch after treatment & 3 days \\
\hline 034 & C-DEA 2x 2h & Itch after treatment & $1-2$ days \\
\hline 051 & C-DEA 2x 2h & Itch during shampooing off & 3-4 minutes \\
\hline 057 & C-DEA 2x 2h & Itch during shampooing off & 3-4 minutes \\
\hline
\end{tabular}

